\title{
PREVALENCIA DE ALTERACIONES URODINÁMICAS EN LAS INFECCIONES URINARIAS RECIDIVANTES DE LA MUJER
}

Jesús Salinas, Miguel Tellez', Miguel Virseda², Juan Carlos Ramírez y Ángel Silmi.

Servicio Urología. Hospital Clínica San Carlos- Universidad Complutense. Madrid.

'Servicio Urología. Hospital Severo Ochoa. Leganés. Madrid.

${ }^{2}$ Servicio Urología. Hospital Central de la Defensa. Madrid. España.

Resumen.- OBJETIVO: Comprobar los datos urodinámicos en las pacientes con infecciones urinarias recidivantes en orden a demostrar una causa que las justifique.

MÉTODOS: Se realiza un estudio transversal comparativo en una serie de 114 mujeres, de edad media 51.9 años (desviación típica: 23.5 años), divididas en dos grupos de 57 mujeres cada uno: grupo con infecciones urinarias recidivantes (ITU), y grupo sin infecciones urinarias recidivantes (no ITU). A las pacientes se les sometió a interrogatorio clínico, exploración física y estudio videourodinámico. Los datos fueron almacenados en una base de datos Access y posteriormente importados al programa de análisis estadísticos SPSS. Se aplicaron el test exacto de Fisher, Chi-Cuadrado de Pearson, y t de Student. Se calculó la curva ROC. Se elaboró un modelo multivalente de regresión logística.

RESULTADOS: Únicamente se demostraron diferencias significativas en los valores del flujo miccional máximo (menor en el grupo ITU), volumen residuo postmiccional (mayor en el grupo ITU), y presión de la contracción involuntaria del detrusor (menor en el grupo ITU). No obstante, la única variable independiente fue el residuo postmiccional, constituyéndose las dos restantes en variables dependientes. El punto de corte ideal entre residuo postmiccional e infección urinaria correspondió a un residuo de $48.5 \mathrm{ml}$. El coeficiente de determinación del modelo fue de 0.13. No se demostraron relaciones significativas de las infecciones urinarias con lentre otras): la presencia y grado de cistocele, así como con la hiperactividad del detrusor e incontinencia urinaria de esfuerzo.

CONCLUSIONES: El residuo postmiccional explicaría el $13 \%$ de las infecciones urinarias recidivantes de la mujer. El resto se debería a otros factores no incluidos en el modelo.

Palabras clave: Urodinámica. Infecciones urinarias recidivantes. Mujer.

Summary.- OBJECTIVES: To check the urodynamic data in patients with recurrent urinary tract infection in order to demonstrate a cause justifying them.

METHODS: We performed a transverse comparative study in a series of 114 women, with a mean age of 51.9 yr. (typical deviation: 23.5 yr.), divided into groups of 57 women each: group I with recurrent urinary tract 
infection (UTI) and group II without recurrent urinary tract infection (no UTI). Patients underwent history, physical examination, and video-urodynamic study. All data were collected in an Access database and subsequently imported to the SPSS statistical analysis software. Fisher's exact test, Pearson's chi-square, and Student's t-test were applied. ROC curve was calculated. A logistic regression multivalue model was elaborated.

RESULTS: significant differences were only found in the values of maximum voiding flow (lower in the UTI group), post void residual volume (greater in the UTI group), and pressure of the involuntary detrusor contraction llower in the UTI group). Nevertheless, post void residual was the only independent variable, becoming the other two dependent variables. The ideal cut point between post void residual and urinary tract infection was $48.5 \mathrm{ml}$. The determination coefficient for the model was 0.13. No significant relationships were found between urinary tract infection and, among others, presence and degree of cystocele, detrusor hyperactivity, and stress urinary incontinence.

CONCLUSIONS: Postroid residual would explain 13\% of the recurrent urinary tract infection in women. The remainder would be secondary to other factors not included in the model.

Keywords: Urodynamics. Recurrent urinary tract infection. Female.

\section{INTRODUCCIÓN}

El sexo femenino presenta una incidencia de infecciones urinarias 10 veces superior al masculino. Un $20 \%$ de las mujeres experimentan infecciones del tracto urinario inferior a la largo de su vida, y aproximadamente el $3 \%$ tienen infecciones recidivantes del tracto urinario, considerando éstas como tres o más episodios al año (1).

Los estudios urodinámicos en las infecciones urinarias recurrentes o recidivantes han sido descritas sobre todo en la edad infantil (2-5) y menos frecuentes en la mujer (6), no alcanzándose conclusiones claras.

El objetivo de nuestro trabajo ha sido realizar un estudio comparativo sobre la prevalencia de alteraciones urodinámicas en mujeres con infecciones urinarias recidivantes, frente a otro grupo de mujeres, sin infecciones urinarias recidivantes.

\section{MATERIAL Y MÉTODOS}

Se realizó un estudio transversal analítico en una serie de 114 mujeres, de edad media 51, 9 años (desviación típica: 23,5 años), divididas en dos grupos de 57 mujeres en cada uno: grupo de mujeres con motivo fundamental de consulta de infecciones urinarias recidivantes (ITU), y grupo comparativo sin infecciones urinarias recidivantes (no ITU).

Los criterios para incluir a las pacientes en el grupo ITU fueron: más de 3 episodios de infección urinaria/año. Se excluyeron las vejigas neurógenas y malformaciones del tracto urinario.

La selección de las pacientes de ambos grupos se efectuó de manera sucesiva.

Las pacientes fueron sometidas a un interrogatorio clínico sobre la presencia de síntomas de la fase de llenado y miccional. A continuación se efectuó una exploración física registrándose la existencia de cistocele, rectocele o prolapso uterino y midiéndolos según una escala ordinal de 0 a 4 .

Por último se realizó un estudio urodinámico que consistió en:

a). Una flujometría libre con medida del flujo máximo expresado en $\mathrm{ml} / \mathrm{s}$; volumen miccional, expresado en $\mathrm{ml}$, residuo postmiccional expresado en $\mathrm{ml}$; y presencia de actividad EMG durante la micción, (medida de forma dicotómica).

b). Una cistomanometría con medida de la capacidad vesical expresada en $\mathrm{ml}$; presión detrusor al final del llenado, expresada en $\mathrm{cm} \mathrm{H}_{2} \mathrm{O}$; presencia de contracciones involuntarias del detrusor (medida en forma dicotómica), y la presión de la contracción involuntaria, expresada en $\mathrm{cm} \mathrm{H}_{2} \mathrm{O}$.

c). Un estudio presión flujo con medida de la presión máxima del detrusor; presión del detrusor correspondiente al flujo máximo lambas expresadas en $\mathrm{cm} \mathrm{H}_{2} \mathrm{O}$ ); flujo miccional máximo expresado en $\mathrm{ml} / \mathrm{s}$, URA ("Urethral Resistance Averaged") expresada en $\mathrm{cm} \mathrm{H}_{2} \mathrm{O}$; Wmax y $\mathrm{W}_{80-20}$ (ambas expresadas en Watios $/ \mathrm{m}^{(2)}$; actividad EMG durante la micción y micción con prensa abdominal (ambas medidas dicotómicamente).

d). Un estudio videourodinámico, valorándose los siguientes parámetros: incontinencia urinaria de esfuerzo (dicotómica), cistocele radiológico (escala ordinal con cinco categorías: ausencia, grado 1, 2, 3 y 4), apertura cuello vesical durante la micción (dicotómica), reflujo vesicoureteral (escala ordinal con cuatro 
categorías: ausencia, grado 1, 2 ó 3) y lateralidad del reflujo vesicoureteral (escala nominal con tres categorías: derecha, izquierda, bilateral).

Los datos fueron almacenados en una base de datos ACCESS y posteriormente importados al programa de análisis estadístico SPSS. El análisis estadístico se realizó aplicando el test exacto de Fisher para variables dicotómicas, el test de la chi-cuadrado de Pearson para la comparación de variables categóricas y el test de comparación de medias de la t de Student para la comparación variables paramétricas. El punto de corte de máxima sensibilidad y especificidad se calculó mediante la construcción de una curva ROC. El análisis de la independencia de las variables predictivas se realizó mediante la elaboración de un modelo multivariante de regresión logística. El nivel de significación se fijo en el $95 \%$ bilateral.

\section{RESULTADOS}

\section{Estadística descriptiva}

La edad media del grupo ITU fue de 51,93 (desviación típica: 23,42). La edad media del grupo no ITU fue de 51,84 (desviación típica: 23, 48). Las diferencias no fueron significativas $(p=0,984)$

La distribución de los síntomas urinarios se muestra en la Tabla I. El síntoma que con más frecuencia referían las pacientes fue la incontinencia con la tos $(53 \%$ de la muestra), seguido de la nicturia (46 $\%)$, y la urgencia-incontinencia (44\%).

La distribución de los datos exploratorios se muestra en la Tabla II. No se comprobó ningún caso de rectocele y sólo un caso de prolapso uterino que fue grado III. En el $67 \%$ de las pacientes tampoco se observó cistocele.

La distribución de los datos flujométricos se muestran en la Tabla III. La de los datos cistomanométricos en la Tabla IV y la de los datos de presión/ flujo en la Tabla V. La distribución de los datos videourodinámicos se muestran en la Tabla VI.

\section{Estadística analítica}

\section{Estadistica bivariante}

La comparación entre el grupo de pacientes con ITU y no ITU, respecto a la distribución de síntomas urinarios se muestra en la Tabla VII. Se observó que las pacientes del grupo no ITU referían en un porcentaje significativamente mayor, incontinencia urinaria con la tos e urgencia-incontinencia.

La comparación entre el grupo de pacientes con ITU y no ITU, respecto al grado de cistocele se muestra en la Tabla VIII. Las diferencias no fueron significativas.

La comparación entre el grupo de pacientes con ITU y no ITU, respecto a los datos flujométricos

TABLA I. DISTRIBUCIÓN DE LOS SÍNTOMAS URINARIOS.

\begin{tabular}{|l|l|l|l|}
\hline Síntoma & Presente & Ausente & Total \\
\hline Nicturia & $52(46 \%)$ & $62(54 \%)$ & 114 \\
\hline Polaquiuria & $49(43 \%)$ & $65(57 \%)$ & 114 \\
\hline Urgencia miccional & $10(9 \%)$ & $104(91 \%)$ & 114 \\
\hline Urgencia incontinencia & $50(44 \%)$ & $64(56 \%)$ & 114 \\
\hline Dificultad miccional & $32(28 \%)$ & $82(72 \%)$ & 114 \\
\hline Calibre miccional disminuido & $25(22 \%)$ & $89(78 \%)$ & 114 \\
\hline Micción intermitente & $9(8 \%)$ & $105(92 \%)$ & 114 \\
\hline Goteo terminal & $1(\%)$ & $113(99 \%)$ & 114 \\
\hline Incontinencia con la tos & $60(53 \%)$ & $54(47 \%)$ & 114 \\
\hline
\end{tabular}


TABLA II. DISTRIBUCIÓN DE LOS DATOS EXPLORATORIOS.

\begin{tabular}{|l|l|l|l|l|l|}
\hline & Ausencia & Grado I & Grado II & Grado III & Total \\
\hline Cistocele & $76(67 \%)$ & $30(26 \%)$ & $7(6 \%)$ & $1(1 \%)$ & 114 \\
\hline Rectocele & $114(100 \%)$ & 0 & 0 & 0 & 114 \\
\hline Prolapso uterino & $113(99 \%)$ & 0 & 0 & $1(1 \%)$ & 114 \\
\hline
\end{tabular}

TABLA III. DISTRIBUCIÓN DE DATOS FLUJOMÉTRICOS.

\begin{tabular}{|l|l|}
\hline Flujo miccional máximo $(\mathrm{ml} / \mathrm{s})^{*}$ & $18,9(9,87)$ \\
\hline Volumen miccional $(\mathrm{ml}) *^{*}$ & $267,79(184,87)$ \\
\hline Residuo postmiccional $(\mathrm{ml})^{*}$ & $34,85(74,58)$ \\
\hline Actividad EMG durante la micción† & $60(53 \%)$ \\
\hline
\end{tabular}

* Media (entre paréntesis desviación típica), † frecuencia absoluta (entre paréntesis porcentaje)

\section{TABLA IV. DISTRIBUCIÓN DE DATOS CISTOMANOMÉTRICOS.}

\begin{tabular}{|l|l|}
\hline Capacidad vesical $(\mathrm{ml})^{*}$ & $215,79(109,43)$ \\
\hline Presión vesical de llenado $\left(\mathrm{cm} \mathrm{H}_{2} \mathrm{O}\right)^{*}$ & $6,00(6,85)$ \\
\hline Contracciones involuntarias $\dagger$ & $46(40 \%)$ \\
\hline Presión máxima de la contracción involuntaria $\left(\mathrm{cm} \mathrm{H}_{2} \mathrm{O}\right)^{*}$ & $61,37(63,62)$ \\
\hline
\end{tabular}

* Media (entre paréntesis desviación típica), † frecuencia absoluta (entre paréntesis porcentaje)

\section{TABLA V. DISTRIBUCIÓN DE LOS DATOS DE PRESIÓN/ FLUJO.}

\begin{tabular}{|l|l|}
\hline Presión máxima del detrusor $\left(\mathrm{cm} \mathrm{H}_{2} \mathrm{O}\right)^{*}$ & $35,90(29,24)$ \\
\hline Presión detrusor a flujo máximo $\left(\mathrm{cm} \mathrm{H}_{2} \mathrm{O}^{*}\right.$ & $23,96(17,83)$ \\
\hline Flujo máximo $(\mathrm{ml} / \mathrm{s})^{*}$ & $13,22(13,64)$ \\
\hline URA $\left(\mathrm{cm} \mathrm{H} \mathrm{H}_{2}\right)^{*}$ & $14,17(11,91)$ \\
\hline Wmax $\left(\text { Watios } / \mathrm{m}^{2}\right)^{*}$ & $13,31(13,64)$ \\
\hline $\mathrm{W}_{80-20}\left(\text { Watios } / \mathrm{m}^{2}\right)^{*}$ & $1,50(3,53)$ \\
\hline Micción con prensa† & $72(63 \%)$ \\
\hline Actividad EMG durante la micción† & $73(64 \%)$ \\
\hline
\end{tabular}

* Media (entre paréntesis desviación típica), † frecuencia absoluta (entre paréntesis porcentaje) 


\section{TABLA VI. DISTRIBUCIÓN DE LOS DATOS VIDEOURODINÁMICOS.}

\begin{tabular}{|l|lr|}
\hline Incontinencia de esfuerzo* $^{|l|} 18$ (16\%) \\
\hline Grado cistocele* $^{|l|}$ Ausencia: $30(26 \%)$ \\
Grado I: & $5(4 \%)$ \\
& Grado II & $24(21 \%)$ \\
& Grado III & $50(44 \%)$ \\
& Grado IV & $5(4 \%)$ \\
\hline Reflujo vesicoureteral ${ }^{*}$ & Ausencia $107(94 \%)$ \\
& Grado I: & $3(3 \%)$ \\
& Grado II: & $2(2 \%)$ \\
& Grado III & $2(2 \%)$ \\
\hline Lateralidad reflujo* $^{*}$ & Ausencia $107(94 \%)$ \\
& Izquierdo: & $2(2 \%)$ \\
& Derecho & $2(2 \%)$ \\
Bilateral & $3(3 \%)$ \\
\hline
\end{tabular}

* frecuencia absoluta (entre paréntesis porcentaje)

se muestran en la Tabla IX. Se observaron diferencias significativas respecto al flujo miccional máximo y el residuo postmiccional, en el sentido de un menor flujo miccional máximo, y mayor residuo postmiccional en el grupo ITU.

La comparación entre el grupo de pacientes con ITU y no ITU, respecto a los datos cistomanométricos se muestran en la Tabla X. Se observaron diferencias significativas respecto a la presión del detrusor durante la contracción involuntaria.

La comparación entre el grupo de pacientes con ITU y no ITU, respecto a los datos de presión/ flujo se muestran en la Tabla XI. No se observaron diferencias significativas.

La comparación entre el grupo de pacientes con ITU y no ITU, respecto a los datos videourodinamicos se muestran en la Tabla XII. No se observaron diferencias significativas.

La curva de rendimiento diagnóstico respecto a la relación entre residuo postmiccional e infec- ción urinaria se muestra en la Figura 1. El punto de corte ideal correspondió a un residuo postmiccional de $48,5 \mathrm{ml}$.

\section{Estadística multivariante}

Los resultados del análisis de regresión logística multivariante se muestran en la Tabla XIII y XIV. En el modelo inicial se incluyeron las tres variables predictoras que fueron significativas en el análisis bivariante (flujo miccional máximo, presión de contracción involuntaria y residuo postmiccional). Mediante el método de eliminación por pasos, se demostró que la única variable predictora independiente fue el residuo postmiccional. El coeficiente de determinación del modelo fue de 0,13 . Es decir el residuo postmiccional explica el $13 \%$ de las infecciones urinarias. El resto se debe a otros factores no incluidos en el modelo.

\section{DISCUSIÓN}

Es de señalar la falta de síntomas específicos del tracto urinario inferior (LUTS) en las infecciones urinarias recidivantes de la mujer, referidas siempre al intérvalo entre infecciones. Ésto es, no en el pe-

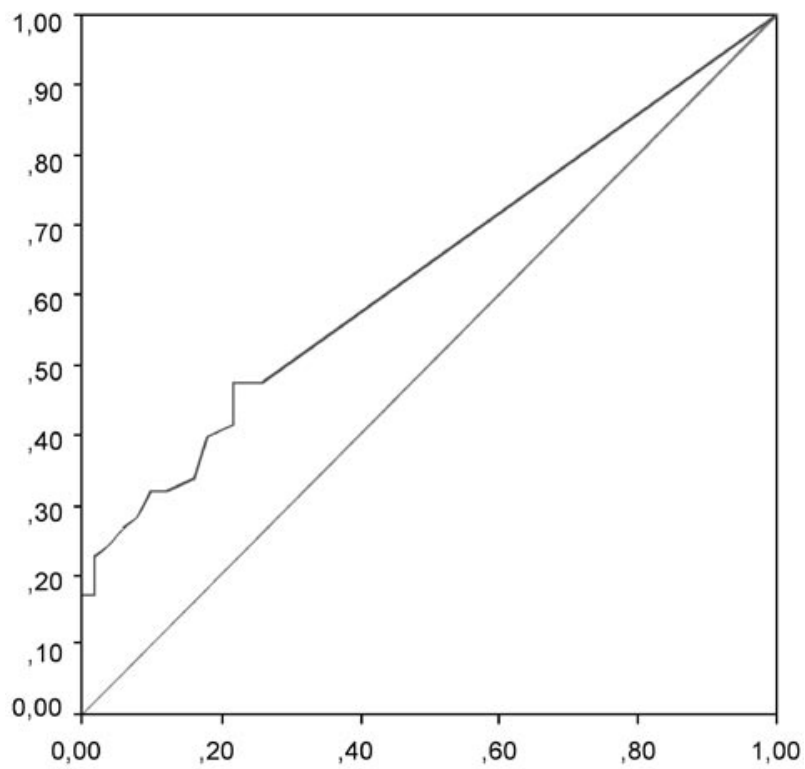

\section{1 - Especificidad}

Los segmentos diagonales son producidos por los empates.

FIGURA 1. Curva ROC del residuo postmiccional respecto a la presencia de infecciones urinarias recidivantes. 
TABLA VII. RELACIÓN SINNTOMAS URINARIOS/INFECCIONES URINARIAS RECIDIVANTES.

\begin{tabular}{|l|l|l|l|}
\hline Síntoma* & ITU & No ITU & Nivel de significación \\
\hline Nicturia & $27(47 \%)$ & $25(44 \%)$ & 0,851 \\
\hline Polaquiuria & $27(47 \%)$ & $22(39 \%)$ & 0,449 \\
\hline Urgencia miccional & $8(14 \%)$ & $2(3 \%)$ & 0,094 \\
\hline Urgencia incontinencia & $19(33 \%)$ & $54(31 \%)$ & $\dagger 0,037$ \\
\hline Dificultad miccional & $14(25 \%)$ & $18(32 \%)$ & 0,532 \\
\hline Calibre miccional disminuido & $14(25 \%)$ & $11(19 \%)$ & 0,651 \\
\hline Micción intermitente & $5(9 \%)$ & $4(7 \%)$ & 1 \\
\hline Goteo terminal & $0(0 \%)$ & $1(2 \%)$ & 1 \\
\hline Incontinencia con la tos & $20(35 \%)$ & $40(70 \%)$ & $\dagger 0,000$ \\
\hline
\end{tabular}

* Presencia en frecuencia absoluta (entre paréntesis porcentaje), † significativo.

ríodo agudo de la infección urinaria. Es más, en el grupo no ITU, se demostró un porcentaje mayor del síntoma incontinencia urinaria, tanto a la tos, como urgencia-incontinencia, frente al grupo ITU. Este dato explica porqué en el grupo ITU, el motivo fundamental de consulta son las infecciones urinarias recidivantes, mientras que el grupo no ITU, representaría el grupo comparativo, sin infecciones urinarias recidivantes, $y$ donde la incontinencia urinaria es más frecuente. $Y$ aparte, sin olvidar cierto sesgo de la muestra.

TABLA VIII. RELACIÓN GRADO CISTOCELE/ INFECCIONES URINARIAS RECIDIVANTES.

\begin{tabular}{|l|l|l|l|l|l|}
\hline & Ausencia & Grado I & Grado II & Grado III & Total \\
\hline ITU & $39(68 \%)$ & $15(26 \%)$ & $3(5 \%)$ & $0(0 \%)$ & 114 \\
\hline No ITU & $37(63 \%)$ & $15(26 \%)$ & $4(7 \%)$ & $1(1 \%)$ & 114 \\
\hline
\end{tabular}

$\chi^{2}$ Pearson $p=0,754$

TABLA IX. RELACIÓN DATOS FLUJOMÉTRICOS /INFECCIONES URINARIAS RECIDIVANTES.

\begin{tabular}{|l|l|l|l|}
\hline Flujo miccional máximo $(\mathrm{ml} / \mathrm{s})^{*}$ & ITU & No ITU & significación \\
\hline Volumen miccional $(\mathrm{ml})^{*}$ & $17,06(8,67)$ & $20,96(10,72)$ & $\ddagger 0.046$ \\
\hline Residuo postmiccional $(\mathrm{ml})^{*}$ & $274,30(204,53)$ & $260,88(163,26)$ & 0,713 \\
\hline Actividad EMG durante la micción† & $55,40(95,96)$ & $13,08(29,45)$ & $\ddagger 0,004$ \\
\hline
\end{tabular}

* Media (entre paréntesis desviación típica), † frecuencia absoluta (entre paréntesis porcentaje) ‡ significativo. 
TABLA X. RELACIÓN DATOS CISTOMANOMÉTRICOS/INFECCIONES URINARIAS RECIDIVANTES.

\begin{tabular}{|l|l|l|l|}
\hline & ITU & No ITU & Significación \\
\hline Capacidad vesical $(\mathrm{ml})^{*}$ & $224,57(119,17)$ & $207,02(99,04)$ & 0,398 \\
\hline Presión vesical de llenado $\left(\mathrm{cm} \mathrm{H}_{2} \mathrm{O}\right)^{*}$ & $5,32(3,92)$ & $6,68(8,54)$ & 0,282 \\
\hline Contracciones involuntarias† & $26(46 \%)$ & $20(35 \%)$ & 0,337 \\
\hline $\begin{array}{l}\text { Presión máxima de la contracción } \\
\text { involuntaria }\left(\mathrm{cm} \mathrm{H}_{2} \mathrm{O}\right)^{*}\end{array}$ & $15,68(19,94)$ & $34,73(15,68)$ & $\ddagger 0,046$ \\
\hline
\end{tabular}

* Media (entre paréntesis desviación típica), † frecuencia absoluta (entre paréntesis porcentaje) † significativo.

Algunos autores (7) han descrito un aumento de la incidencia de las infecciones urinarias en la mujer, de un $5 \%$ en edad fértil, a un $10 \%$ después de la menopausia, atribuyendo entre otros factores, al déficit estrogénico. En este dato, se apoyaría el tratamiento hormonal sustitutivo en las infecciones urinarias recidivantes en dicho período de edad (8).

En nuestro trabajo, no se pueden extraer datos en este sentido, al tratarse de un estudio transversal comparativo.

En nuestra serie, los prolapsos pélvicos, no fueron muy frecuentes, no demostrándose ningún caso de prolapso del comportamiento posterior (rectocele), y un solo caso de prolapso uterino grado III. Se observó cistocele en el $33 \%$ de los casos, pero no se observó ninguna relación significativa (tanto la presencia, como grado de cistocele, de la exploración física y videourodinámica) con las infecciones urinarias. Este dato no apoyaría la corrección quirúrgica del cistocele, como tratamiento dirigido a erradicar las infecciones urinarias recidivantes de la mujer.

Respecto al estudio urodinámico de la flujometría, si se demostró en nuestra serie, relación significativa entre el valor del flujo miccional máximo y residuo postmiccional, con las infecciones urinarias. En el grupo ITU se demostró un flujo miccional máximo disminuido, y un mayor volumen de residuo postmiccional,

TABLA XI. RELACIÓN DATOS DE PRESIÓN/FLUJO/INFECCIONES URINARIAS RECIDIVANTES.

\begin{tabular}{|l|l|l|l|}
\hline & ITU & No ITU & significación \\
\hline Presión máxima del detrusor $\left(\mathrm{cm} \mathrm{H}_{2} \mathrm{O}\right)^{*}$ & $57(38,35)$ & $56(33,41)$ & 0,371 \\
\hline Presión detrusor a flujo máximo $\left(\mathrm{cm} \mathrm{H}_{2} \mathrm{O}\right)^{*}$ & $24,98(16,81)$ & $22,91(18,91)$ & 0,540 \\
\hline Flujo máximo $(\mathrm{ml} / \mathrm{s})^{*}$ & $12,3(7,29)$ & $14,16(11,79)$ & 0,316 \\
\hline URA $\left(\mathrm{cm} \mathrm{H} \mathrm{H}_{2}\right)^{*}$ & $15,26(12,19)$ & $13,05(11,61)$ & 0,326 \\
\hline$W_{m a x}\left(\mathrm{Watios} / \mathrm{m}^{2}\right)^{*}$ & $14,13(13,78)$ & $12,46(13,57)$ & 0,519 \\
\hline $\mathrm{W}_{28-20}\left(\mathrm{Watios} / \mathrm{m}^{2}\right)^{*}$ & $1,76(3,67)$ & $1,23(3,39)$ & 0,426 \\
\hline Micción con prensa $\dagger$ & $35(61,4 \%)$ & $37(64,9 \%)$ & 0,846 \\
\hline Actividad EMG durante la micción $\dagger$ & $38(67 \%)$ & $35(61 \%)$ & 0,697 \\
\hline
\end{tabular}

* Media (entre paréntesis desviación típica), † frecuencia absoluta (entre paréntesis porcentaje). 
TABLA XII. RELACIÓN DATOS VIDEOURODINÁMICOS/INFECCIONES URINARIAS RECIDIVANTES.

\begin{tabular}{|c|c|c|c|}
\hline & ITU & No ITU & Significación \\
\hline Incontinencia de esfuerzo* & $57(38,35)$ & $56(33,41)$ & 0,371 \\
\hline Grado cistocele* & $\begin{array}{c}\text { Ausencia: } 14 \text { (24\%) } \\
\text { I: } 1(2 \%) \\
\text { II: } 11(19 \%) \\
\text { III: } 29(51 \%) \\
\text { IV : } 2(3 \%)\end{array}$ & $\begin{array}{c}\text { Ausencia: } 14(24 \%) \\
\text { I:4 (7\%) } \\
\text { II: } 13(23 \%) \\
\text { III: } 21(37 \%) \\
\text { IV: } 3(5 \%)\end{array}$ & 0,466 \\
\hline Reflujo vesicoureteral* & $\begin{array}{c}\text { Ausencia: } 53(93 \%) \\
\text { I: } 1(2 \%) \\
\text { II: } 2(3 \%) \\
\text { III: } 1(2 \%)\end{array}$ & $\begin{array}{c}\text { Ausencia: } 55(96 \%) \\
\begin{array}{c}\text { I: } 1(2 \%) \\
\text { II: } 0(0 \%) \\
\text { III: } 1(2 \%)\end{array}\end{array}$ & 0,565 \\
\hline Lateralidad reflujo* & $\begin{array}{l}\text { Izdo: } 1(2 \%) \\
\text { Dcho: } 2(3 \%) \\
\text { Bil.. } 2(3 \%)\end{array}$ & $\begin{array}{l}\text { Izdo: } 1(2 \%) \\
\text { Dcho: } 0(0 \%) \\
\text { Bil.. } 1(1 \%)\end{array}$ & 0,490 \\
\hline
\end{tabular}

* Media (entre paréntesis desviación típica).

TABLA XIII. RESULTADOS DEL ANÁLISIS DE REGRESIÓN MULTIVARIANTE. MODELO INICIAL.

\begin{tabular}{|l|c|c|c|c|}
\hline & Coeficiente de regresión & Desviación típica & Significación & $\begin{array}{c}\text { Coeficiente de } \\
\text { determinación }\end{array}$ \\
\hline Residuo postmiccional & 0,0110 & 0,0052 & 0,038 & 0,14 \\
\hline Flujo máximo & $-0,0339$ & 0,0226 & 0,1332 & \\
\hline Presión contracción & $-0,0079$ & 0,0053 & 0,1399 & \\
\hline Constante & 0,6084 & 0,5249 & 0,2465 & \\
\hline
\end{tabular}

TABLA XIV. RESULTADOS DEL ANÁLISIS DE REGRESIÓN LOGÍSTICA MULTIVARIANTE. MODELO FINAL.

\begin{tabular}{|l|c|c|c|c|}
\hline & Coeficiente de regresión & Desviación típica & Significación & $\begin{array}{c}\text { Coeficiente de } \\
\text { determinación }\end{array}$ \\
\hline Residuo postmiccional & 0,0131 & 0,0051 & 0,0190 & 0,13 \\
\hline Constante & $-0,2767$ & 0,2313 & 0,2316 & \\
\hline
\end{tabular}


frente a grupo no ITU. No obstante el flujo miccional máximo se comportó en el estudio multivariante como una variable dependiente del residuo postmiccional.

El punto de corte ideal de la curva de rendimiento diagnóstico (curva Roc) en la relación entre residuo postmiccional e infección urinaria, correspondió a un residuo postmiccional de $48,5 \mathrm{ml}$. El coeficiente de determinación del medio fue de 0,13 . Ésto es, el residuo postmiccional explicaría el $13 \%$ de las infecciones urinarias recidivantes. El $87 \%$ restante se debería a otros factores.

Este dato tendría mucha trascendencia, ya que de esto se desprendería, que ante infecciones urinarias recidivantes en la mujer, sólo la demostración de un residuo postmiccional mayor de $48,5 \mathrm{ml}$., podría sospechar la existencia de una disfunción vesicouretral, y justificar la realización de un estudio urodinámico completo.

Por otra parte, en estos casos no habría que alcanzar un volumen residual de $100 \mathrm{ml}$. o el 10-20 $\%$ del volumen miccional para considerarlo como un residuo significativo (9).

Esteban y cols. (10) en su serie de incontinencia urinaria en la mujer, encontraron que las pacientes con residuo postmiccional presentaron bacteriuria significativa en el $26.3 \%$ de los casos.

Aunque la medición del residuo postmiccional puede hacerse por cateterismo vesical, cistografía, "bladder scan" (11), la más aconsejable sería la ecografía abdominal, aprovechando la ecografía urológica habitual (renal y vesical) que se realiza a este tipo de enfermas. Esto es, la medida ecográfica del residuo postmiccional debe incluirse en la realización de la ecografía renal/vesical en las enfermas con infecciones urinarias recidivantes.

En la determinación ecográfica del residuo postmiccional, se realiza la medida de los diámetros vesicales, anteroposterior transverso y cefalocaudal, aplicando la fórmula del elipsoide $V=$ $0.57 \times D 1 \times D 2 \times D 3$ (11).

Aunque los valores del flujo miccional máximo de la flujometría, podrían ayudarnos al estar disminuidos significativamente en el grupo ITU, no sería necesario realizar este test en este tipo de enfermas, al ser una variable dependiente del residuo postmiccional (que es la variable independiente).

La medida del residuo postmiccional se convertiría así en la técnica de "screening" en las infecciones urinarias recidivantes de la mujer.
No se ha demostrado en nuestro trabajo relación significativa entre las infecciones urinarias recidivantes y la hiperactividad del detrusor, a pesar de que Rees (12) describe un $25 \%$ de hiperactividad del detrusor en cistitis recidivantes, y Moore y cols. (13) encuentra un asociación significativa entre hiperactividad del detrusor y cistitis bacteriana. Por el contrario, Esteban y cols. (10), tampoco demuestran una relación estadística en la incontinencia urinaria femenina entre la urgencia-incontinencia e hiperactividad del detrusor por una parte, y la bacteriruria significativa por otra parte. En este sentido coinciden Okorocha y Cumming (14), que no encuentran asociación entre la hiperactividad del detrusor y la infección urinaria.

Lapides y Costello (15) describen que presiones elevadas del detrusor, sobre todo, en forma de contracciones involuntarias del detrusor comprimirían la pared vesical, resultando en isquemia, que favorecería la cistitis bacteriana.

Esta hipótesis no ha sido comprobada en nuestra serie, ya que no se demostraron mayores presiones de las contracciones involuntarias del detrusor en el grupo ITU, así como tampoco en la revisión de Walter y cols. (16).

Es más, en nuestro trabajo se ha demostrado unas menores presiones de las contracciones involuntarias del detrusor en el grupo ITU, que podrían explicarse por una afectación muscular vesical por su exposición a las endotoxinas del E-Coli (17).

Tampoco se ha demostrado en nuestra muestra una relación significativa entre el diagnóstico videourodinámico de incontinencia urinaria de esfuerzo e infecciones urinarias de repetición, como algunos autores han demostrado. Mommsen y cols. (18), describieron cistitis en el $63 \%$ de los pacientes con incontinencia urinaria de esfuerzo aislada, frente al $56 \%$ de las pacientes con urgencia-incontinencia. Así mismo, Bergman y cols. (19) obtienen la continencia urinaria en el $30 \%$ de las pacientes con incontinencia urinaria de esfuerzo que habían sido sometidas a tratamiento antibiótico de su infección urinaria.

Por el contrario, Okorocha y Cumming (14) coinciden con nuestros datos, al no encontrar asociación entre la incontinencia urinaria de esfuerzo y la infección urinaria.

Finalmente, la demostración videourodinámica de reflujo vesicoureteral en nuestra serie no presentó relación significativa con las infecciones urinarias recidivantes, dato frecuentemente descrito en la edad infantil (20). 


\section{BIBLIOGRAFÍA Y LECTURAS RECOMENDADAS ( ${ }^{*}$ lectura de interés $y^{* *}$ lectura fundamental)}

1. PONTONES MORENO, J.L.; MOLA ARIZO, M.J.; JIMÉNEZ CRUZ, J.F.: "Profilaxis antimicrobiana en Urología”. Chap. 5 p. 75-86. En Patología Urológica Infecciosa. Chap 5 p75-86. Ed S. Navío- Pub Aula Médica. Madrid. 1999.

2. SHAIK, N.; ABEDIN, S.; DOCIMO, S.G.: "Can ultrasonography or uroflowmetry predict which children with voiding dysfunction will have recurrent urinary tract infections?". J. Urol., 174: 1620, 2005.

3. VAN GOOL, J.; TANAGHO, E.A.: "External sphincter activity and recurrent urinary tract infection in girls". Urology, 10: 348, 1977.

4. CHANDRA, M.; MADDIX, H.; Mc VICAR, M.: "Transient urodynamic dysfunction of infancy: relationship to urinary tract infections and vesicoureteric reflux". J. Urol., 155: 673, 1996.

5. KOFF, S.A.; LAPIDES, J.; PIAZZA, D.H.: “Association of urinary tract infection and reflux with uninhibited bladder contractions and voluntary sphincter obstruction". J. Urol., 122: 373, 1979.

6. WALTER, S.; ANDERSEN, J.T.; HEBSORN, S. y cols.: "Detrusor hyperreflexia and bacteriuria". Urol. Int., 32: 117, 1977.

7. LACY, S.S.: "Urinary tract infection. Gynecologic and Obstetric Urology". Ed by HS Buchsbaum, JD Schmidt. Philadelphia W-B. Saunders, pp. 301-324, 1978.

8. JIMÉNEZ CRUZ, J.F; VERA DONOSO, C.D.: "Infección urinaria de repetición en la mujer". EU: Jiménez Cruz, J.F. (ed): Infección Urinaria. Barcelona. Doyma, 57-64, 1991.

9. SALINAS, J.; VIRSEDA, M.: "Flujometría y medida del residuo postmiccional". En controversias en urodinámica. I. Introducción a la urodinámica. p57-58. Ed J. Salinas y M. Virseda. Pub. Entheos. Madrid. 2006.
10. ESTEBAN, M.; SALINAS, J.; RAPARIZ, M. y cols.: "Incontinencia urinaria y bacteriuria en la mujer”. Arch. Esp. Urol., 47: 591, 1994.

*11. SALINAS, J.; SÁNCHEZ, E.; VIRSEDA, M. y cols.: "Fiabilidad de la estimación del volumen vesical postmiccional mediante ultrasonidos. Comparación de dos métodos ecográficos". Arch. Esp. Urol., 49: 35, 1996.

12. REES, D.L.: "Urinary tract infection. Clinics in Obstetrics and Gynecology". Ginecology Urology, Ed. by S.L. Stanton. London. W.B. Saunders, pp. 169-193, 1978.

*13. MOORE, K.H.; SIMONS, A.; MOSERKEE, C. y cols.: "The relative incidence of detrusor instability and bacterial cystitis detected on the urodinamic-test day". BJU International, 85: 786, 2000.

*14. LAPIDES, J.; COSTELLO, R.T.: "Uninhibited neurogenic bladder: A common cause for recurrent urinary infection in normal women". J. Urol., 101: 539, 1969.

15. WALTER, S.; ANDERSEN, J.T.; HEBJORN, S. y cols.: "Detrusor hyperreflexia and bacteriuria". Urol. Int., 32: 117, 1977.

16. NERGARDH, A.; BOREUS, L.O.; HOLE, T.: "The inhibitory effect of coli-endotoxin on alphaadrenergic receptor function in the lower urinary tract. An in vitro study in cats". Scand. J. Urol. Nephrol., 11: 219, 1977.

17. MOMMSEN, S.; FOLDSPANG, A.; ELVING, L. y cols.: "Cystitis as a correlate of female urinary incontinence". Int. Urogynecol. J., 5: 135, 1994.

**18. BERGMAN, A.; NARENDER, N.; BHATIA, N.: "Urodynamics: Effect of urinary tract infection on urethral and bladder function". Obstet. Gynecol., 66: 366, 1985.

19. KOFF, S.A.; WAGNER, T.T.; SAYANTHI, V.R.: "The relationship among dysfunctional elimination syndromes, primary vesicoureteral reflux and urinary tract infections in children". J. Urol., 160: 1019, 1998.

20. OKOROCHA, I.; CUMMING, G.: "Female urodynamics and lower urinary tract infection". BJU Int., 89: 863, 2002. 